\title{
Structural Evolution of a Stimulus-responsive Diblock Polypeptide Micelle by Temperature Tunable Compaction of its Core
}

Elisabeth Garanger, ${ }^{\dagger}, \star$ Sarah R. MacEwan, ${ }^{\S}$ Olivier Sandre, ${ }^{\dagger}$ Annie Brûlet, ${ }^{,}$Laure Bataille, ${ }^{\dagger,}$ Ashutosh Chilkoti, ${ }^{\circledR}$ Sébastien Lecommandoux ${ }^{* \dagger}$

† Laboratoire de Chimie des Polymères Organiques (LCPO), CNRS UMR 5629, Université de Bordeaux, Bordeaux-INP, Pessac 33607 Cedex, France.

${ }^{*}$ Institut Européen de Chimie et Biologie (IECB), Pessac 33607, France.

$\S$ Department of Biomedical Engineering, Campus Box 90281, Duke University, Durham, North Carolina 27708, United States.

${ }^{\swarrow}$ Laboratoire Léon Brillouin (LLB), CEA-CNRS UMR 12, CEA-Saclay, Gif-sur-Yvette 91191, France.

*Fax: 3354000 8487; Tel: 3354000 3035; E-mail: e.garanger@iecb.u-bordeaux.fr

* Fax: 1919660 5409; Tel: 1919660 5373; E-mail: chilkoti@duke.edu

*Fax: 3354000 8487; Tel: 3354000 2241; E-mail:lecommandoux@enscbp.fr 


\section{Abstract}

With a perfectly defined primary structure, both in terms of monomer sequence and chain length, recombinant polypeptides obtained by protein engineering techniques allow the investigation of structure-property relationships at a level of detail that is difficult to achieve with traditional synthetic polymers because of the precision with which their sequence can be defined. In the present work, we have studied the behavior and temperature-triggered self-assembly of a series of diblock recombinant elastin-like polypeptides (ELPs) with the goal of elucidating the mechanism of their self-assembly into micelles. Aqueous solutions of diblock ELPs were studied below and above their critical micellar temperature (CMT) by multi-angle light scattering and small-angle neutron scattering techniques. Below the $C M T$, the radius of gyration of soluble ELP chains follows a power law as a function of molecular weight with an exponent value close to 0.5 that is characteristic of Gaussian coil conformations. As the temperature reaches the $C M T$, attractive interactions between the more hydrophobic block of diblock ELP chains leads to the self-assembly of monodisperse spherical micelles at thermodynamic equilibrium. Above the $C M T$, micelles expel water molecules from their core whose densification is evidenced by the monotonic increase in the light and neutron scattering intensities as a function of temperature. The behaviors of these different diblock ELPs in solution and as self-assembled nanoparticles above the CMT following universal experimental scaling laws make them analogous to synthetic amphiphilic diblock copolymers (star-like vs. crew-cut micelle models). These studies also shed light on the important role of water in the thermal behavior of these thermally-responsive self-assembling diblock polypeptides and suggest a new design parameter - thermally-triggered desolvation and densification of the core of micelles - that can be fine tuned at the sequence level to control the density of self-assembled polymer nanoparticles. 


\section{INTRODUCTION}

Elastin-like polypeptides (ELPs) have recently attracted significant attention as precision protein-like polymers and constitute an attractive alternative to synthetic polypeptides obtained by traditional polymerization processes. ${ }^{1,2}$ Recombinant DNA and protein-engineering techniques used to produce them inherently lead to macromolecules with perfectly defined primary sequence and chain length, and allow the incorporation of relevant peptide or protein domains with specific biological activity. ${ }^{3}$ For example, ELP block copolymers have recently been engineered to serve as a platform for the multivalent presentation of small peptides and single domain proteins onto ELP-based nanoparticles. ${ }^{4}$

ELPs are biopolymers of -Val-Pro-Gly-Xaa-Gly- pentapeptides, thereafter noted (VPGXG), derived from native tropoelastin (the guest residue at the fourth position - Xaa - being any amino acid except proline). ${ }^{5} \mathrm{~A}$ defining property of ELPs in aqueous solution is their phase transition upon raising the temperature above a critical temperature, namely the lower critical solution temperature (LCST, also called inverse transition temperature, noted as $T_{\mathrm{t}}$ ). Below the LCST, ELP chains are fully hydrated and soluble, but upon raising the solution temperature above the LCST, ELP chains hydrophobically collapse into insoluble aggregates to form a coacervate (i.e. demixed) liquid phase containing 63\% water and $37 \%$ polymer by weight. $6,7 \mathrm{~A}$ number of parameters have been shown to directly affect the LCST value including macromolecular parameters such as polypeptide molecular weight, composition (in particular the nature of the guest residue) and ELP architecture, as well as environmental parameters such as the type and concentration of co-solutes and pH. ${ }^{5 b, 8 ; 9,10}$

ELP block copolymers (ELPBCs) result from the linear and covalent association of two or more ELP segments with different LCST values. Above a critical micellization temperature (CMT), as the "hydrophobic block" has desolvated independently of the more hydrophilic block(s), ELP $P_{\mathrm{BCs}}$ form spherical micelles with a core composed of the hydrophobic ELP block and a corona composed of the hydrophilic ELP block(s). These micelles are stable over a specific temperature range, precisely up to a second threshold temperature (i.e., LCST of diblock ELP) above which the micelles macroscopically aggregate. ${ }^{11,12}$ The mean polarity of ELP $_{\mathrm{BCs}}$, the spatial arrangement of amino acids and 
distribution of polar and apolar domains along the ELP chains have been shown to impact the transition temperature of individual blocks. ${ }^{13}$

While general trends were proposed to describe the LCST and temperature-induced selfassembly of polymers, the complex role of water molecules on hydration and conformation of polymer chains is unclear, especially in the case of protein-like polymers that might have both the characteristics of synthetic polymers ${ }^{14,15}$ and proteins. ${ }^{16,17} \mathrm{~A}$ recent study by Hinderberger and coworkers ${ }^{18}$ has shown, using continuous wave (CW) electron paramagnetic resonance (EPR) spectroscopy, that two kinds of hydration layers (respectively ascribed to the hydrophilic backbone and the hydrophilic residues) exist in ELP homopolymers in either a coupled or a decoupled state that are functions of the ELP primary sequence. The authors demonstrated that the decoupled state denotes individual temperature-dependent dehydration of the two types of hydration layers found in ELPs, while the coupled state is characterized by a common, cooperative phase transition of both associated water layers.

The present work was motivated by peculiar features in the temperature-triggered selfassembly of diblock ELPs into micelles. It was previously seen that as the temperature of a diblock ELP solution is increased above the CMT, the diblock ELP gains sufficient amphiphilicity to undergo self-assembly into spherical micelles. ${ }^{12}$ Raising the temperature further yields to a plateau region in which the micelles exhibit colloidal stability, with a constant hydrodynamic size. However, for certain diblocks, a continuous increase of the scattered light intensity was observed in this plateau regime of size, the origins of which are unclear. To understand this behavior, a series of recombinant diblock ELPs with different hydrophobic block sizes was designed and produced, and the structural characteristics of the resulting nanoparticles were determined as a function of temperature using light and neutron scattering techniques. (Figure 1) These studies yielded the unexpected observation that once formed above the $C M T$, micelles are first strongly hydrated, and then continue to evolve thermally. A further increase in temperature leads to a progressive compaction of their core through a temperaturedriven desolvation of the core-forming ELP segment. To our knowledge, this temperature-triggered core compaction is observed for the first time for a diblock ELP 
copolymer system providing a new structural variable that can be explored in the design of recombinant self-assembling block copolymers.

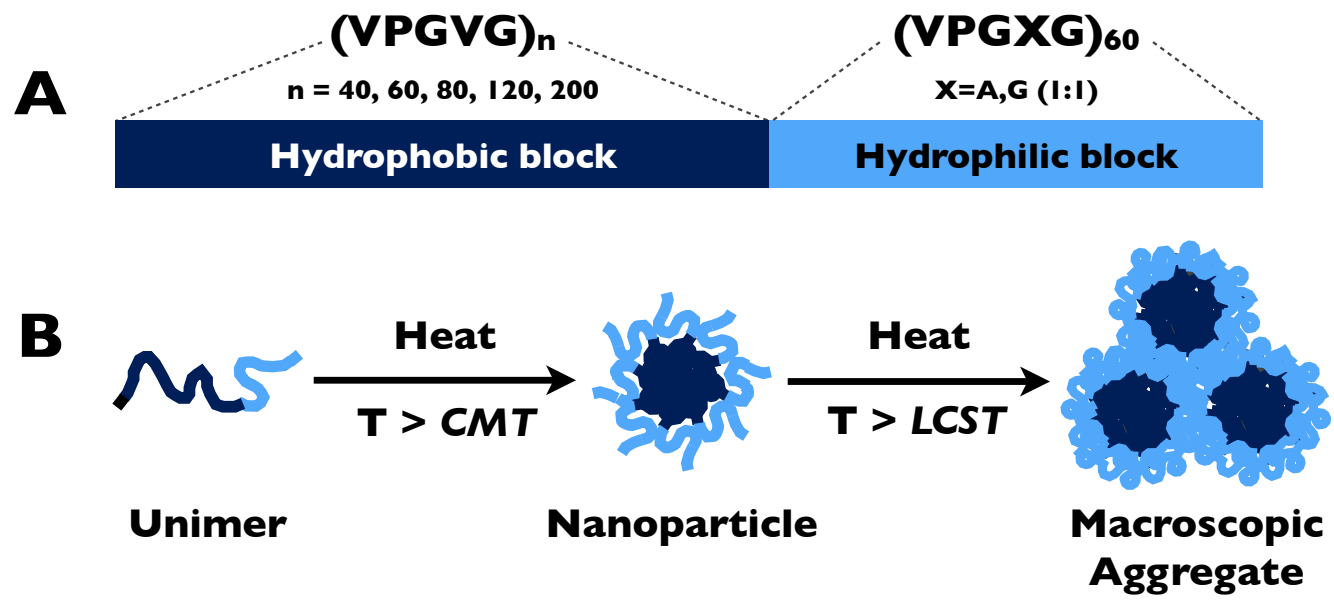

Figure 1. Schematic representation of diblock ELPs' sequence (A), their self-assembly above the CMT and aggregation above the LCST (B).

\section{EXPERIMENTAL SECTION}

\section{Gene Synthesis}

Synthetic genes for the diblock ELPs were assembled from chemically synthesized oligonucleotides by recursive directional ligation by plasmid reconstruction (PReRDL). ${ }^{19}$ This cloning method allows modular and seamless assembly of ELP genes, and enables easy construction of genes encoding diblock ELPs with peptide functionalization at their $N$ - and $C$-termini. Each diblock ELP gene encoded a hydrophobic domain with the sequence $(V G V P G)_{n}(n=40,60,80,120$, or 200$)$ and a hydrophilic domain with the sequence (AGVPGGGVPG) 30 . A MGCGWPG peptide sequence (termed "leader") was encoded at the $N$-terminus proximal to the hydrophobic ELP domain, and a PGGS peptide sequence (termed "trailer") was encoded at the $C$-terminus proximal to the hydrophilic ELP domain. Exact nucleotide sequences of diblock ELP-encoding genes and amino acid sequences of diblock ELPs are provided in the supplementary information (ESI) accompanying the manuscript. ELP genes were constructed in a pET-24a(+) cloning vector encoding antibiotic resistance to kanamycin using EB5Alpha competent E. coli (Edge BioSystems, Gaithersburg, MD). Successful cloning was confirmed by DNA sequencing with $\mathrm{T} 7$ promoter and $\mathrm{T} 7$ terminator primers. 


\section{Diblock ELP Expression and Purification}

Ultra BL21 competent E. coli cells (Edge BioSystems, Gaithersburg, MD) were transformed with the plasmids and grown for $24 \mathrm{~h}$, while shaking at $37^{\circ} \mathrm{C}$, in Terrific Broth (TB) medium containing kanamycin $\left(45 \mu \mathrm{g} \cdot \mathrm{mL}^{-1}\right)$. E. coli cells were collected by centrifugation at 3,200 RPM at $4^{\circ} \mathrm{C}$ and lysed by sonication. Genomic and phospholipid contaminants were condensed by the addition of $10 \% \mathrm{w} / \mathrm{v}$ poly(ethylenimine) and insoluble cellular debris was removed by centrifugation at 14,000 RPM at $4^{\circ} \mathrm{C}$. Soluble ELP in the supernatant was then purified by inverse transition cycling (ITC),20 a nonchromatographic technique that exploits the LCST behavior of the ELP. The ELP transition was triggered with heat $\left(\sim 37^{\circ} \mathrm{C}\right)$ and the addition of crystalline $\mathrm{NaCl}(\sim 1.5$ M). An ELP pellet was collected by centrifugation at 14,000 RPM at a temperature above the ELP transition temperature, while soluble contaminants in the supernatant were discarded. The ELP pellet was resolubilized in cold phosphate buffered saline (PBS, 1.05 $\mathrm{mM} \mathrm{KH}_{2} \mathrm{PO}_{4}, 3 \mathrm{mM} \mathrm{Na}_{2} \mathrm{HPO}_{4}, 154 \mathrm{mM} \mathrm{NaCl}, \mathrm{pH}$ 7.4) and centrifuged at 14,000 RPM at $4^{\circ} \mathrm{C}$ to pellet insoluble contaminants. ELP in the supernatant was retained and subjected to additional rounds of ITC. Repeating steps of centrifugation above and below the ELP transition temperature removed residual soluble and insoluble contaminants, respectively. Approximately four rounds of ITC produced pure diblock ELP products, as confirmed by SDS-PAGE and $\mathrm{CuCl}_{2}$ staining. (ESI, Figure S1) As it is typical of many ELPs, these diblock ELPs migrated with an apparent molecular weight $\left(M_{\mathrm{w}}\right)$ up to $20 \%$ larger than their expected $M_{\mathrm{w}} \cdot{ }^{9 \mathrm{a}, 21}$ Diblock ELPs were also analyzed by Matrix-Enhanced Laser Desorption Ionization (MALDI) mass spectrometry to experimentally confirm their $M_{\mathrm{w}}$ values. (ESI, Table S1) All diblock ELPs are designated as "ELP $n-m$ " where $n$ and $m$ refer to the number of pentapeptide repeats in the $N$-terminal (hydrophobic) and $C$-terminal (hydrophilic) blocks, respectively.

\section{Transition Temperature Measurement}

Transition temperatures $\left(T_{\mathrm{t}}\right)$, that are also often referred to as LCST, were determined by turbidity measurements at $350 \mathrm{~nm}$ between 20 and $80^{\circ} \mathrm{C}$ at $1^{\circ} \mathrm{C} \cdot \mathrm{min}^{-1} \mathrm{scan}$ rate of diblock ELPs in aqueous solution at different concentrations $(5,10,25,50,100 \mu \mathrm{M})$. Data were collected on a Cary 300 Bio UV-visible spectrophotometer equipped with a multi-cell thermoelectric temperature controller (Varian, Palo Alto, CA). The $T_{\mathrm{t}}$ is defined as the temperature corresponding to the maximum of the first derivative of the 
turbidity versus temperature curve. A typical graph of turbidity as a function of temperature for ELP80-60 is provided in the supplementary information. (ESI, Figure S2)

\section{Average Size by Dynamic Light Scattering Intensity Measurements}

Dynamic light scattering (DLS) measurements were performed on a NanoZS instrument (Malvern, UK) at a $173^{\circ}$ angle at a constant position in the cuvette (constant scattering volume). The derived count rate was defined as the mean scattered intensity normalized by the attenuation factor. Three independent measurements of ten 10 s-runs were recorded and averaged. Temperature was raised from 20 to $70^{\circ} \mathrm{C}$ and measurements were performed every degree after a 2 min-temperature equilibration time. Solutions of ELP80-60 were prepared at $0.6,1.1,5.7$ and $11.3 \mathrm{mg} \cdot \mathrm{mL}^{-1}$ concentration $(10,20,100$ and $200 \mu \mathrm{M}$, respectively) in PBS and filtered through $0.22 \mu \mathrm{m}$ cellulose acetate filters before analysis.

\section{Multi-angle Dynamic and Static Light Scattering (DLS, SLS)}

Multi-angle dynamic and static light scattering experiments were performed using an ALV CGS-3 Compact Goniometer System, equipped with a $35 \mathrm{~mW}$ HeNe linearly polarized laser tube of wavelength $632.8 \mathrm{~nm}$ and an ALV/LSE-5004 light scattering electronic and Multiple Tau Digital correlator. The accessible scattering angles ranged from $30^{\circ}$ to $150^{\circ}$. The ELP solutions were prepared at $1.4 \mathrm{mg} \cdot \mathrm{mL}^{-1}$ concentration in PBS and filtered through $0.22 \mu \mathrm{m}$ cellulose acetate filters before analysis. Samples $(4.5 \mathrm{~mL}$ in $2 \mathrm{~cm}$-diameter cylindrical glass cells) were immersed in a filtered toluene bath and equilibrated for $15 \mathrm{~min}$ at each temperature between 30 and $50^{\circ} \mathrm{C}$. Static data of 3 independent 5 s-measurements with less than 3\% standard variation were recorded with the ALV-Correlator Control software. Three independent $20 \mathrm{~s}$-measurements were recorded for dynamic data. Hydrodynamic radii $\left(R_{\mathrm{H}}\right)$ were calculated from the diffusion coefficient $(D)$ using the Stokes-Einstein relation:

$$
D=\frac{k_{\mathrm{B}} T}{6 \pi \eta_{\mathrm{S}} R_{\mathrm{H}}}
$$

Eq. 1

where $D$ is calculated from the slope of the curve $1 / \tau$ as a function of $q^{2} . \tau$ is the main correlation time determined by the $2^{\text {nd }}$ order cumulant analysis of the autocorrelogram, $q$ the scattering vector defined as $q=(4 \pi / \lambda) \sin (\theta / 2)$ with $\lambda$ the wavelength and $\theta$ the 
scattering angle, $k_{\mathrm{B}}$ the Boltzmann constant, $T$ the temperature in Kelvin and $\eta_{\mathrm{S}}$ the viscosity of the solvent.

Radii of gyration $\left(R_{\mathrm{G}}\right)$ were determined from the plot of $\ln I(q)$ as a function of $q^{2}$ using the Guinier approximation:

$$
\ln I(q)=\ln I(0)-\frac{q^{2}{R_{\mathrm{G}}}^{2}}{3}
$$

Eq. 2

where $R_{\mathrm{G}^{2}}{ }^{2} / 3$ corresponds to the slope of the curve.

Nanoparticle apparent molar weights $\left(M_{\mathrm{w}}{ }^{*}\right)$ were obtained from the inverse of the ordinate at origin of a linear regression of $K \cdot c / R(q)$ as a function of $q^{2}+k \cdot c$, where $q$ is the scattering vector, $c$ the weight concentration and $k$ an arbitrary multiplicative factor in the so-called Kratky plots. $R$ is the Rayleigh ratio defined as:

$$
R(q)=\frac{I(q)-I_{s}(q)}{I_{\mathrm{tol}}(q)} \cdot \frac{n_{\mathrm{s}}^{2}}{n_{\mathrm{tol}}^{2}} R_{\mathrm{tol}}
$$

Eq. 3

The previously determined refractive index increment $\frac{d n}{d C}=0.1750 \mathrm{~mL} \cdot \mathrm{g}^{-1}$ (with an Optilab $^{\text {TM }}$ T-rEX differential refractometer, Wyatt Technology Corp., Santa Barbara, CA) was used to compute the $K$ scattering coefficient:

$$
K=\frac{2}{N_{A} \cdot \lambda^{4}}\left(\pi \cdot n_{\mathrm{s}} \cdot \frac{d n}{d C}\right)^{2}
$$

Eq. 4

where $I(q), I_{\mathrm{s}}(q)$ and $I_{\mathrm{tol}}(q)$ are the count-rates measured by the detector at an angle $\theta$ corresponding to the scattering vector $q$ for respectively sample, solvent and toluene. $R_{\mathrm{tol}}$ is the Rayleigh ratio of toluene at $\lambda=632.8 \mathrm{~nm}\left(R_{\mathrm{tol}}=1.4 \times 10^{-3} \mathrm{~m}^{-1}\right) .{ }^{22}$ The tabulated values of the refraction indexes were also used, respectively $n_{S}=1.333$ for water and $n_{\mathrm{tol}}=1.491$ for toluene.

\section{Small Angle Neutron Scattering (SANS)}

SANS measurements were performed on the PACE spectrometer of the Laboratoire Léon Brillouin (CEA-Saclay, France). ELP solutions were prepared in fresh $\mathrm{D}_{2} \mathrm{O}$ at $5 \mathrm{mg} \cdot \mathrm{mL}^{-1}$ for the study of individual chains at $15^{\circ} \mathrm{C}$ or $10 \mathrm{mg} \cdot \mathrm{mL}^{-1}$ for the study of aggregation as a function of temperature. For the diluted samples at $15^{\circ} \mathrm{C}$, three configurations were used to cover overlapping $q$ ranges of $2.4 \times 10^{-3}-2.6 \times 10^{-2}, 6.9 \times 10^{-3}-7.3 \times 10^{-2}$, and $3.6 \times 10^{-2}-$ $0.37 \AA^{-1}$, with the following values of sample-to-detector distance $D$ and neutron 
wavelength $\lambda: D=4.57 \mathrm{~m}$ and $\lambda=17 \AA, D=4.57 \mathrm{~m}$ and $\lambda=6 \AA, D=0.87 \mathrm{~m}$ and $\lambda=6 \AA$. For measurements of the more concentrated solutions at increasing temperature, the small angles and large angles configurations were the same as for the experiments at $15^{\circ} \mathrm{C}$, but the intermediate angles configuration was $D=2.86 \mathrm{~m}$ and $\lambda=6 \AA$ covering a $q$ range of $1.1 \times 10^{-2}-1.2 \times 10^{-1} \AA^{-1}$.

\section{Modeling of SANS data}

Analysis of the scattering intensity aims at obtaining the characteristic sizes, the shape and the interactions, represented by the form factor $P(q)$ and the structure factor $S(q)$. Numerous models have been proposed to describe the scattering by density fluctuations of colloidal or polymeric systems. ${ }^{23}$ Classical expression of the scattering intensity per unit volume of spherically symmetric particles writes:

$$
I(q)=n \Delta \rho^{2} V_{\text {part. }}^{2} P(q) S(q)
$$

\section{Eq. 5}

where $n$ is the number density of particles, $\Delta \rho$ is the difference in the neutron scattering length density between the particles and the solvent, and $V_{\text {part. }}$ is the unit volume of the particles. The form factor describes the structure of particles and fulfills $P(q=0)=1$ while the structure factor describes the interaction between particles. In the absence of interactions, $S(q)=1$. Introducing the volume fraction of particles, $\Phi=n V_{\text {part. }}$ Eq. 5 becomes:

$$
I(q)=\Phi \Delta \rho^{2} V_{\text {part. }} P(q)
$$

Eq. 6

For a sphere with radius $R$, the form factor writes: ${ }^{24}$

$$
P_{\text {sphere }}(q, R)=\left(\frac{3[\sin (q R)-q R \cos (q R)]}{(q R)^{3}}\right)^{2}
$$

Eq. 7

Modeling of individual ELP chains (unimers) at low temperature (below the CMT)

In order to describe the behavior of ELPs at low temperature, we focused on polymer chain models rather than on geometrical form factors as for particles. For individual chains, the volume $V_{\text {part. }}$ is defined by the weight average molecular weight $M_{\mathrm{w}}$ of one mole of chains, the molar mass $m$ and the volume $v$ of one monomer as $V_{\text {chain }}=$ 
$M_{\mathrm{w}} \cdot v / m$. Thus, for a dilute solution of polymer of weight concentration $c$, occupying a volume fraction $\Phi=N_{\mathrm{A}} \cdot v \cdot c / m$, where $N_{\mathrm{A}}$ is the Avogadro number, Eq. 6 becomes:

$$
I(q)=v^{2} \Delta \rho^{2} \frac{c}{m^{2}} N_{\mathrm{A}} M_{\mathrm{w}} P(q)
$$

Eq. 8

By introducing the mass density of the polymer $d=m /\left(N_{\mathrm{A}} \cdot v\right)$, we obtain:

$$
I(q)=\Delta \rho^{2} \frac{c}{d^{2} N_{\mathrm{A}}} M_{\mathrm{w}} P(q)
$$

Eq. 9

Generally, the weight average molecular weight $M_{\mathrm{w}}$ and the radius of gyration $R_{\mathrm{G}}$ can be deduced from the fit to this equation using the so called Debye function ${ }^{25}$ as form factor:

$$
P_{\text {Debye }}\left(q, R_{\mathrm{G}}\right)=\frac{2}{\left(q^{2} R_{\mathrm{G}}^{2}\right)^{2}}\left(\exp \left(-q^{2} R_{\mathrm{G}}^{2}\right)+q^{2} R_{\mathrm{G}}^{2}-1\right)
$$

Eq. 10

Modeling of self-assembled ELP chains above the CMT

To fit the SANS signal above the CMT of the diblock ELPs, the self-assembled structures were represented by spherical particles interacting through the hard-sphere (excluded volume) interaction potential. The structure factor was calculated in the Percus-Yevick approximation for the closure relationship. ${ }^{26,27}$ The parameters are the hard-sphere radius, $R_{\mathrm{HS}}$, and the volume fraction of hard-spheres, $\eta_{\mathrm{HS}}$. Another slightly different model which could better describe the structure of aggregates of amphiphilic diblock copolymers is the core-shell model with hard-sphere interaction, with the core radius $R_{\mathrm{c}}$ and the shell thickness $\delta$ as parameters, combined with an attractive interaction potential ("stickiness") at the surface. ${ }^{28,29}$. However, the simpler model of hard spheres was sufficient to fit the SANS data of the micelles formed by diblock ELPs. The fits were achieved with the SasView program (http://www.sasview.org/) with polydisperse spherical particles or with a customized program written in house for the individual polymer chains. Size distribution and instrument resolution with appropriate weighting were taken into account in all the fitting procedures, ${ }^{23}$ as done previously for another type of peptide-polymer micelles of narrow size-dispersity. ${ }^{30}$

\section{RESULTS AND DISCUSSION}

Diblock ELPs design, production and characterization 
A series of five diblock ELPs was designed containing a hydrophilic ELP block of fixed size at the $C$-terminal end of the polypeptide and a more hydrophobic ELP block of variable length at the $N$-terminal end so as to cover a large range of hydrophilic weight fractions. (Figure 1A) The hydrophilic block, designed to have a high transition temperature (above $60^{\circ} \mathrm{C}$ ), contained 60 repeats of (VPGXG) pentapeptides, the guest residue $\mathrm{X}$ being alternatively alanine (A) or glycine (G). ${ }^{19}$ The hydrophobic block contained valine (V) at the X position of the (VPGXG) repeats. The (VPGVG) was varied from $n=40$ to 200 repeats, leading to global hydrophilic weight fractions ranging from 21 to $57 \% .{ }^{31}$ (Table 1) For the reader's convenience, we adopt here the same nomenclature as proposed by Chilkoti and coworkers ${ }^{12}$ to designate each diblock ELP: "ELPn- $m$ " where $n$ and $m$ refer to the number of pentapeptide repeats in the $N$ terminal (hydrophobic) and $C$-terminal (hydrophilic) blocks, respectively. The genes encoding for the full diblock ELP sequences were constructed using Recursive Directional Ligation by Plasmid Reconstruction (PRe-RDL), ${ }^{19}$ and cloned in Escherichia coli. The BL21 E. coli strain was used for the expression of diblock ELPs that were subsequently recovered and purified by an inexpensive, non-chromatographic process termed Inverse Transition Cycling (ITC). ${ }^{20,21}$ After extensive dialysis and lyophilization, their purity and molecular weight were assessed by sodium docecyl sulfate polyacrylamide gel electrophoresis (SDS-PAGE) and MALDI mass spectrometry. (ESI, Figure S1 and Table S1) The CMT of the five constructs was measured by absorbance spectroscopy and found, as expected, to decrease with increasing hydrophobic block size, starting from $42^{\circ} \mathrm{C}$ for ELP40-60 with the shortest hydrophobic segment down to $26^{\circ} \mathrm{C}$ for the largest ELP200-60 construct. (Table 1) The LCST of the diblock ELP, resulting from the desolvation of the hydrophilic block leading to macroscopic aggregation of the diblock ELPs, was approximately $65^{\circ} \mathrm{C}$ for all constructs.

Table 1. Diblock ELPs' characteristics.

\begin{tabular}{cccc} 
ELPn-m & $\begin{array}{c}\text { Hydrophobic block } \\
\text { length }(\boldsymbol{n})\end{array}$ & $\begin{array}{c}\text { Hydrophilic weight } \\
\text { fraction }^{\mathrm{a}}(\mathbf{\%})\end{array}$ & $\left.\boldsymbol{C M}^{\mathrm{b}}{ }^{\circ} \mathbf{C}\right)$ \\
\hline $\mathbf{4 0 - 6 0}$ & 40 & 57 & 42 \\
$\mathbf{6 0 - 6 0}$ & 60 & 47 & 34 \\
$\mathbf{8 0 - 6 0}$ & 80 & 40 & 31 \\
$\mathbf{1 2 0 - 6 0}$ & 120 & 31 & 28 \\
$\mathbf{2 0 0 - 6 0}$ & 200 & 21 & 26 \\
\hline
\end{tabular}


a Defined as the ratio of the molecular weight of the hydrophilic block over the molecular weight of the full diblock ELP; ${ }^{b}$ Corresponds to the temperature of self-assembly into micelles due to dehydration of the hydrophobic (VPGVG) $)_{\mathrm{n}}$ sequence. CMTs were determined by the increase of optical absorbance at $350 \mathrm{~nm}$ for a $25 \mu \mathrm{M}$ aqueous solution of diblock ELP.

\section{Initial study of ELP80-60 by dynamic light scattering}

The colloidal behavior in phosphate buffer of ELP80-60 was first examined over a large temperature range and at 4 different concentrations using DLS at a fixed angle $\left(173^{\circ}\right)$. These scouting experiments examined the effect of temperature on the light intensity (derived count rate) scattered by the self-assembled structures, their hydrodynamic radius $\left(R_{\mathrm{H}}\right)$ and their polydispersity index (PDI), as defined by cumulant analysis. This study also addressed specific issues such as heating rates and heating/cooling cycling effects. When monitoring the back-scattered light intensity as well as the size distribution of the particles in solution with gradually increasing temperature, three successive regimes were observed. (Figure 2) 


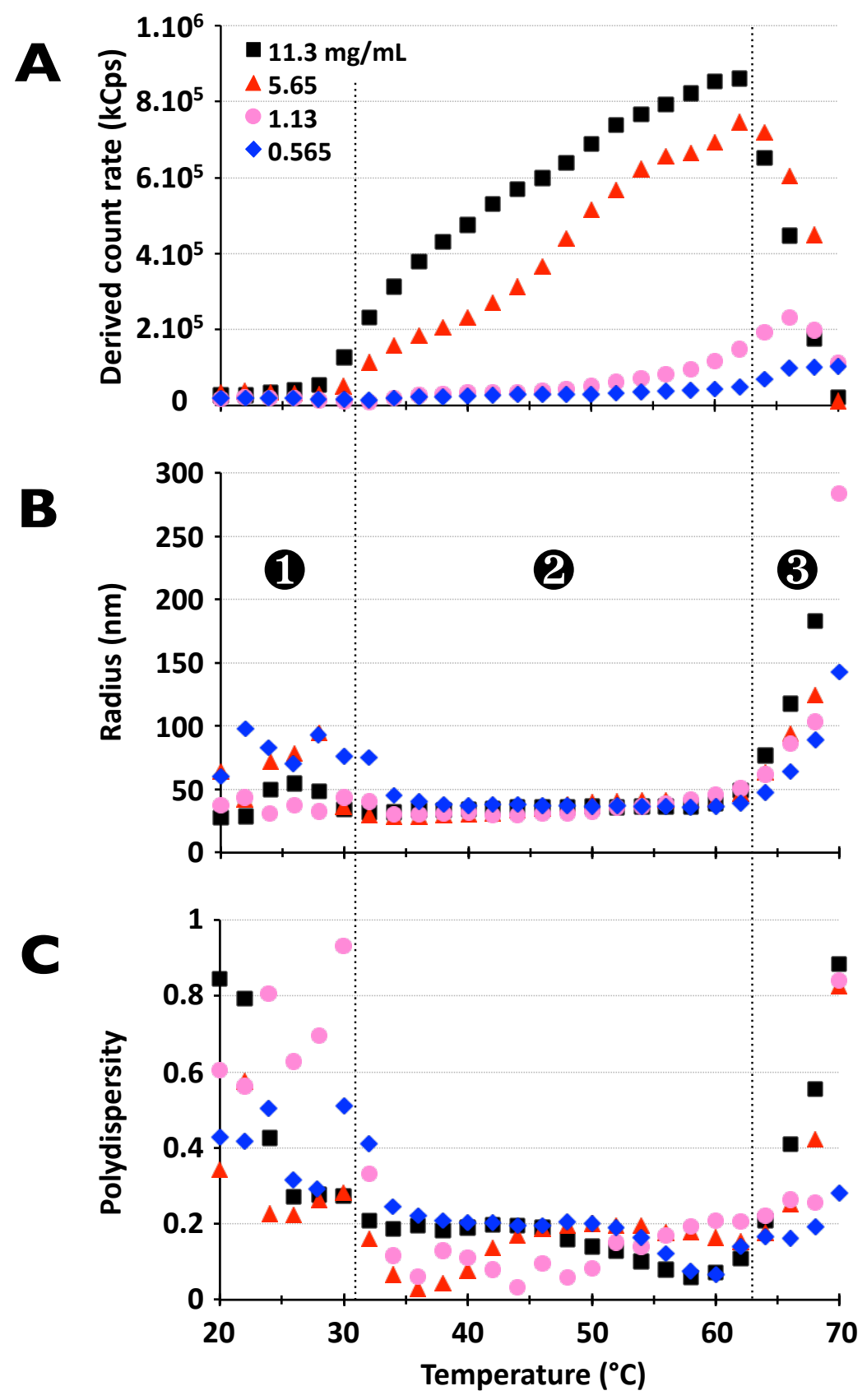

Figure 2. DLS of ELP80-60 diblock in aqueous buffer at different concentrations at $173^{\circ}$. Back-scattered light intensity (A), hydrodynamic radius (B), and polydispersity index (C).

The first regime, below the CMT, was characterized by a low scattered light intensity signal and a high PDI, reflecting mostly weakly scattering ELP single chains coexisting with very few dust particles. Above the CMT and below the LCST of the diblock ELP (second regime), a rather monodisperse population $(\mathrm{PDI}<0.2)$ of nanoparticles was measured with a hydrodynamic radius around $30 \mathrm{~nm}$. The third regime, above the LCST, 
was characterized by a drastic rise in radius and PDI reflecting the macroscopic aggregation of the whole ELP chains as sketched on Figure 1B and the formation of large and polydisperse aggregates that sediment, explaining the associated drop in the derived count rate. Additional experiments reproduced these observations with no effect of heating rate or heating/cooling cycling on all parameters monitored (i.e., derived count rate, $R_{\mathrm{H}}$ and PDI), suggesting that identical self-assembled structures are systematically formed irrespective of the heating conditions. (ESI, Figures S3 and S4) In the nanoparticle regime, the scattered light signal also proved to be stable with increasing time (monitored for up to $3 \mathrm{~h}$ ) at a constant temperature, suggesting that the system has reached thermodynamic equilibrium. (ESI, Figure S5)

However, an intriguing observation was the gradual increase of the scattered light intensity (raw derived count-rate on the detector after conversion as Rayleigh ratio) with increasing temperature over the whole range between the CMT and LCST (nanoparticle regime), suggesting an increase in the scattering contrast of the nanoparticles relative to the solvent. This observation was first reported by Dreher et al.,12 and nanoparticles of this type were classified as type II nanoparticles, but the origin of this behavior had not been investigated. According to Eqs. 2-4, this increase can result from the change in three parameters: (1) the nanoparticles radius of gyration $\left(R_{\mathrm{G}}\right),(2)$ the molecular weight of the scatterers $\left(M_{\mathrm{w}}{ }^{*}\right)$, or (3) the increase in differential refractive index increment $(\mathrm{d} n / \mathrm{d} c)$. The last possibility was eliminated, as very little variation in $\mathrm{d} n / \mathrm{d} c$ values was found for aqueous solutions of ELP80-60 at temperatures ranging from 35 to $50^{\circ} \mathrm{C}$ (ESI). In order to further investigate the origin of this phenomenon, we next carried out DLS and static light scattering (SLS) at multiple angles to enable more precise determination of $R_{\mathrm{H}}$ and $R_{\mathrm{G}}$ in this regime.

\section{Multi-angle dynamic and static light scattering}

The whole set of diblock ELPs was investigated by DLS and SLS, and a similar behavior was observed for all constructs. The detailed angular analyses in dynamic (diffusion coefficient determination) and static mode (Guinier plots) are presented in ESI (Figures S6-10). In the nanoparticle regime (i.e., above CMT and below LCST), $R_{\mathrm{H}}$ was found to slightly increase by a few percent with increasing temperature, while $R_{\mathrm{G}}$ showed no obvious trend. (Table 2) In contrast to $R_{\mathrm{H}}$, which remained nearly constant, the apparent molecular weight $\left(M_{\mathrm{w}}{ }^{*}\right)$ was found to significantly increase with increasing temperature, 
suggesting the formation of self-assembled nanoparticles of greater compactness (i.e., containing more ELP chains in the same volume with an increase in temperature). This however implies either the presence of a reservoir of unassembled ELP chains above the $C M T$, or a reorganization of micelles into objects of similar size but higher density. The second scenario seems more plausible as attractive interactions between the micelles would increase the isotherm compressibility of the suspension $\left(\chi_{\mathrm{T}}\right)^{32}$ and, as a consequence, increase their apparent molecular weight while keeping the radius of gyration quasi-constant. To shed light on these possible scenarios, we next investigated the entire set of diblock ELPs by small angle neutron scattering (SANS) as a function of temperature. However, one has to keep in mind that the contrast is of different origin for light and neutron scattering techniques (respectively the refractive index increment in SLS and the atomic formula neutron scattering cross-section of different atoms in SANS), which makes it difficult to directly compare values of $R_{\mathrm{G}}, M_{\mathrm{W}}{ }^{*}$ and $N_{\mathrm{Agg}}$. obtained by these two techniques although they have an exact definition from polymer physics theory.

Table 2. Structural properties of diblock ELP nanoparticles: hydrodynamic radius $\left(R_{\mathrm{H}}\right)$ and polydispersity index (PDI), radius of gyration $\left(R_{\mathrm{G}}\right)$, and molar weight of the scatterers $\left(M_{\mathrm{w}}{ }^{*}\right)$ as determined by multi-angle dynamic and static light scattering.

\begin{tabular}{|c|c|c|c|c|c|c|}
\hline ELPn-m & Temperature & $30^{\circ} \mathrm{C}$ & $35^{\circ} \mathrm{C}$ & $40^{\circ} \mathrm{C}$ & $45^{\circ} \mathrm{C}$ & $50^{\circ} \mathrm{C}$ \\
\hline & $R_{\mathbf{H}^{\mathrm{a}}}\left(\mathbf{P D I}^{\mathrm{b}}\right)$ & & & & $23.7(0.04)$ & $24.3(0.04)$ \\
\hline \multirow[t]{3}{*}{$40-60$} & $\boldsymbol{R}_{\mathbf{G}^{\mathrm{c}}}$ & & & & 21.4 & 21.1 \\
\hline & $M_{\mathrm{w}}{ }^{* \mathrm{~d}}$ & & & & 4,455 & 5,831 \\
\hline & $\boldsymbol{R}_{\mathrm{H}^{\mathrm{a}}}\left(\mathbf{P D I}^{\mathrm{b}}\right)$ & & & $26.4(0.04)$ & $27.0(0.04)$ & $28.2(0.03)$ \\
\hline \multirow[t]{3}{*}{$60-60$} & $\boldsymbol{R}_{\mathbf{G}^{\mathrm{c}}}$ & & & 20.2 & 20.0 & 20.2 \\
\hline & $M_{\mathrm{w}}{ }^{* \mathrm{~d}}$ & & & 6,625 & 8,359 & 10,520 \\
\hline & $R_{\mathbf{H}^{\mathrm{a}}}\left(\mathrm{PDI}^{\mathrm{b}}\right)$ & & $28.6(0.01)$ & $28.8(0.01)$ & $30.1(0.03)$ & $32.3(0.03)$ \\
\hline \multirow[t]{3}{*}{$80-60$} & $\boldsymbol{R}_{\mathbf{G}^{\mathrm{c}}}$ & & 18.5 & 17.4 & 17.8 & 19.3 \\
\hline & $M_{\mathrm{w}}{ }^{* \mathrm{~d}}$ & & 7,687 & 9,350 & 12,570 & 17,310 \\
\hline & $\boldsymbol{R}_{\mathbf{H}^{\mathrm{a}}}\left(\mathbf{P D I}^{\mathrm{b}}\right)$ & $32.8(0.03)$ & $33.1(0.02)$ & $34.2(0.00)$ & $39.0(0.01)$ & $38.0(0.03)$ \\
\hline \multirow[t]{3}{*}{$120-60$} & $\boldsymbol{R}_{\mathbf{G}^{\mathrm{c}}}$ & 19.8 & 18.3 & 15.5 & 21.3 & 19.5 \\
\hline & $M_{\mathrm{w}}{ }^{* \mathrm{~d}}$ & 9,535 & 13,540 & 17,000 & 31,120 & 30,020 \\
\hline & $R_{\mathbf{H}^{\mathrm{a}}}\left(\mathbf{P D I}^{\mathrm{b}}\right)$ & $45.9(0.07)$ & $45.0(0.08)$ & $47.9(0.04)$ & $49.3(0.06)$ & $54.0(0.07)$ \\
\hline \multirow[t]{2}{*}{$200-60$} & $\boldsymbol{R}_{\mathbf{G}^{\mathrm{c}}}$ & 39.6 & 35.7 & 38.7 & 37.7 & 40.0 \\
\hline & $M_{\mathrm{w}}{ }^{* \mathrm{~d}}$ & 51,520 & 59,280 & 70,140 & 79,280 & 89,900 \\
\hline
\end{tabular}

${ }^{\text {a }} R_{\mathrm{H}}(\mathrm{nm})$ from cumulant analysis; b PDI from cumulant analysis of DLS at a fixed angle of $90^{\circ}$; ${ }^{\mathrm{c}} R_{\mathrm{G}}(\mathrm{nm})$ obtained by SLS (Guinier plots); ${ }^{\mathrm{d}} M_{\mathrm{w}}{ }^{*}\left(\mathrm{~kg} \cdot \mathrm{mol}^{-1}\right)$ obtained by SLS (Berry plots). 
Studies of individual diblock ELP chains (unimers) at low temperature
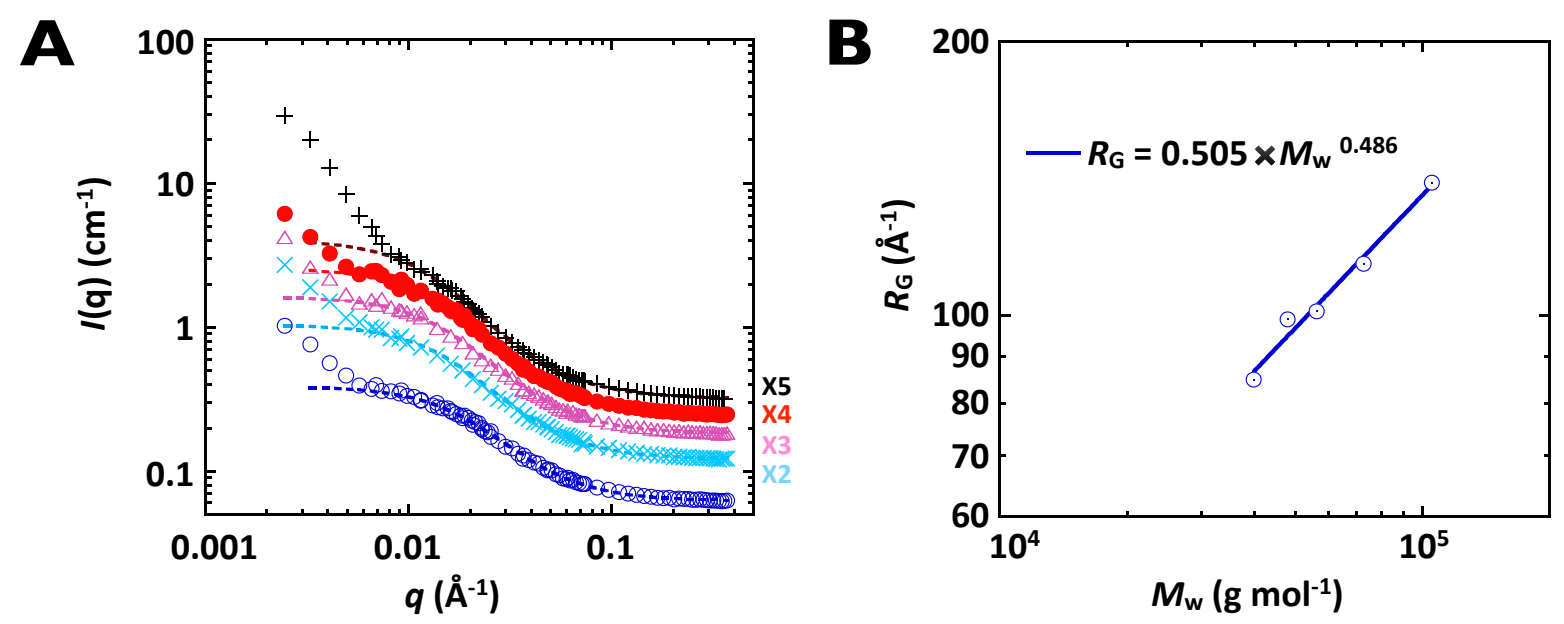

Figure 3. A) SANS curves of dilute solutions of diblock ELPs in $\mathrm{D}_{2} \mathrm{O}$ at $15^{\circ} \mathrm{C}$ : o ELP40-60, x ELP60-60, $\triangle$ ELP80-60, • ELP120-60, + ELP200-60. For better clarity, intensities were shifted by a factor varying from 1 to 5 respectively. Dotted lines are the best fits to the Debye function (best fit parameters are listed in Table 3). B) Scaling law of $R_{\mathrm{G}}$ of diblock ELP chains deduced from Debye fits as a function of their molecular weight $\left(M_{\mathrm{w}}\right)$.

The whole set of diblock ELPs was first studied under dilute conditions at $15^{\circ} \mathrm{C}$, a temperature at which both ELP blocks are solvated. Of course, it is worth mentioning that due to lower bridge-bonding affinity of $\mathrm{D}_{2} \mathrm{O}$ compared to $\mathrm{H}_{2} \mathrm{O}$, slight quantitative differences might be observed compared to DLS analysis. Typical SANS curves are plotted in Figure 3A. Despite the lower concentration used ( $\left.5 \mathrm{mg} \cdot \mathrm{mL}^{-1}\right)$, a significant upturn of the scattering intensity at low $q$ was observed for all diblock ELPs. Being the signature of attractive interactions between individual diblock ELP chains, this signal also increased with the size of the hydrophobic block. However, the physical phenomenon at the origin of this interaction is out of the scope of the present study, since the possible transient clustering of water-soluble chains such as poly(ethylene oxide) (PEO) is a very discussed subject in the literature and still an open question. ${ }^{33} \mathrm{We}$ instead focused on the scattering measured at $q$ higher than $6-8 \times 10^{-3} \AA^{-1}$, corresponding to size scales $2 \pi / q$ below $800 \AA$ A. All curves were well-fit using the Debye function (Eq. 10), indicating that diblock ELP chains behave like linear (Gaussian) polymer chains. Given the known molecular weights of diblock ELPs, and assuming a density $d=1.35 \mathrm{~g}$. $\mathrm{cm}^{-3}$ as reported for proteins in the crystalline state, ${ }^{34}$ best fits to Eqs. 9 and 10 gave the 
$R_{\mathrm{G}}$ of diblock ELP chains as well as the experimental contrast in $\mathrm{D}_{2} \mathrm{O}$. (Table 3) The apparent scattering length densities (SLD), $\rho_{\mathrm{ELP}_{\text {fit }}}$ of the different diblock ELPs were then deduced from their contrast $\Delta \rho_{\text {fit }}$ relative to $\rho_{\mathrm{D}_{2} \mathrm{O}}\left(6.40 \times 10^{10} \mathrm{~cm}^{-2}\right)$.

Table 3. Characteristics of diblock ELP chains in solution in $\mathrm{D}_{2} \mathrm{O}$ measured by SANS at $15^{\circ} \mathrm{C}$.

\begin{tabular}{|c|c|c|c|c|c|c|c|}
\hline \multirow[b]{2}{*}{ ELPn-m } & \multirow[b]{2}{*}{$\begin{array}{c}\rho_{\text {ELP }_{\text {theo }}{ }^{a}}\left(\mathrm{~cm}^{-2}\right)\end{array}$} & \multirow[b]{2}{*}{$\begin{array}{c}c^{\mathrm{b}} \\
\left(\mathrm{g} \cdot \mathrm{cm}^{-3}\right)\end{array}$} & \multicolumn{4}{|c|}{ Debye fit } & \multirow[b]{2}{*}{$N_{/ \mathrm{VPGXG}}^{\mathrm{D}_{2} \mathrm{O}}$} \\
\hline & & & $\begin{array}{l}R_{\mathrm{G} \mathrm{fit}} \\
(\mathrm{nm})\end{array}$ & $\sigma R_{\mathrm{G} \text { fixed }}{ }^{\mathrm{c}}$ & $\begin{array}{c}\Delta \rho_{\mathrm{fit}} \\
\left(\mathrm{cm}^{-2}\right)\end{array}$ & $\begin{array}{l}\rho_{\mathrm{ELP}_{\text {fit }}} \\
\left(\mathrm{cm}^{-2}\right)\end{array}$ & \\
\hline $40-60$ & $1.84 \times 10^{10}$ & 0.00484 & 8.5 & 0.02 & $4.41 \times 10^{10}$ & $1.99 \times 10^{10}$ & 0.6 \\
\hline $60-60$ & $1.82 \times 10^{10}$ & 0.00477 & 9.9 & 0.02 & $4.62 \times 10^{10}$ & $1.78 \times 10^{10}$ & - \\
\hline $80-60$ & $1.80 \times 10^{10}$ & 0.00484 & 10.1 & 0.02 & $4.36 \times 10^{10}$ & $2.04 \times 10^{10}$ & 0.9 \\
\hline $120-60$ & $1.78 \times 10^{10}$ & 0.00511 & 11.4 & 0.02 & $4.34 \times 10^{10}$ & $2.06 \times 10^{10}$ & 1.1 \\
\hline $200-60$ & $1.76 \times 10^{10}$ & 0.00502 & 14.0 & 0.02 & $4.37 \times 10^{10}$ & $2.03 \times 10^{10}$ & 1.0 \\
\hline
\end{tabular}

a Theoretical scattering length densities ( $\rho_{\text {ELP }}{ }_{\text {theo }}$ ) were calculated from the ELP $n-m$ molecular formulas and the scattering lengths of respective atoms, assuming a density $d=1.35 \mathrm{~g} \cdot \mathrm{cm}^{-3}$ to compute the molecular volumes. ${ }^{b}$ Concentrations of diblock ELP solutions measured by gravimetry. ${ }^{c}$ Given the high accuracy of ELPs' molecular weights and their monodispersity, the standard deviation of a Gaussian distribution of $R_{\mathrm{G}}$ was fixed to 0.02 . ${ }^{\mathrm{a}} N_{/ \mathrm{VPGXG}}^{\mathrm{D}_{2} \mathrm{O}}$ is the mean number of $\mathrm{D}_{2} \mathrm{O}$ molecules linked to each pentapeptide that gives the same average SLD as the fit value $\rho_{\mathrm{ELP}}$ fit .

From this series of experimental data, one can analyze the variation of $R_{\mathrm{G}}$ as a function of diblock ELP molecular weight. $R_{\mathrm{G}}$ follows a power law with an exponent value of 0.49 , (Figure 3B) which is characteristic of Gaussian coil conformations $\left(R_{\mathrm{G}}\right.$ scaling as $M_{w}{ }^{0.5}$ for a polymer in $\Theta$-solvent condition). The prefactor of the scaling law leads to a radius of $10 \AA$ for a single pentapeptide of average molecular mass around $400 \mathrm{~g} \cdot \mathrm{mol}^{-1}$, which is in the same order of magnitude than the dimension of a peptide in the dry state (with a density $d=1.35 \mathrm{~g} \cdot \mathrm{cm}^{-3}$ from crystallography, the molecular volume is $500 \AA^{3}$ ). Another analysis of the hydration level arises from the values of scattering length densities deduced from the fits, which were only slightly higher than theoretical values: on average, we found $\rho_{\text {ELP fit }} \sim 2 \times 10^{10} \mathrm{~cm}^{-2}$ instead of $\rho_{\text {ELP theo }}=1.8 \times 10^{10} \mathrm{~cm}^{-2}$ as calculated from the molecular formulas and mass density in the dry state. If ELPs were highly hydrated with $\mathrm{D}_{2} \mathrm{O}$ molecules tightly linked to the backbone, $\rho_{\text {ELP fit }}$ would have been significantly higher than theoretical values $\left(\rho_{\mathrm{ELP}}{ }_{\text {theo }}\right)$ because of the high scattering length density of $\mathrm{D}_{2} \mathrm{O}\left(\rho_{\mathrm{D}_{2} \mathrm{O}}=6.40 \times 10^{10} \mathrm{~cm}^{-2}\right)$. More precisely, one can calculate the number of $\mathrm{D}_{2} \mathrm{O}$ molecules linked to each single ELP chain that would display $\rho_{\text {ELP fit }}$ as 
average SLD: the values $N_{/ V P G X G}^{\mathrm{D}_{2} \mathrm{O}}$ per pentapeptide are listed in Table 3 . Therefore at $15^{\circ} \mathrm{C}$, the hydration of both blocks with only around one $\mathrm{D}_{2} \mathrm{O}$ molecule per pentapeptide reaches the experimental SLD values for the three higher $M_{\mathrm{w}}$ ELPs, and slightly less for the ELPs of lower $M_{\mathrm{w}}$. To summarize these fits of SANS curves of individual ELP chains at low temperature, the analysis of both the dimensions and of the contrast relatively to solvent in terms of hydration levels suggests a weak hydration of ELP chains, even well below the CMT. This result is fully consistent with the Gaussian coil conformation observed, as already inferred for diblock ELP free chains below their CMT from the $q^{-2}$ dependence of their form factor measured by small-angle X-ray scattering. ${ }^{35}$ If the monomers in the ELP chains preferred to be surrounded by solvent molecules rather than with other monomers, the polymer chains would adopt a much more swollen conformation. The variation of $R_{\mathrm{G}}$ as a function of molecular weight would then obey Flory's law, i.e. a power law with an excluded volume exponent of 0.588 , which is not the case with these diblock ELPs, where a value close to 0.5 was calculated, consistent with Gaussian polymer chains in a " $\Theta$-solvent". The largely invariant linear density of 0.6-1.0 $\mathrm{D}_{2} \mathrm{O}$ molecule per pentapeptide for all diblock ELPs is also a direct consequence of the excellent fit of the SANS curves by a Debye form factor that is appropriate for Gaussian linear chains. In the case of other hydrophilic chains exhibiting a LCST behavior such as poly(ethylene oxide) (PEO), the scaling laws of $R_{\mathrm{G}} v s . M_{\mathrm{w}}$ found experimentally vary from 0.58 in good solvent ${ }^{36}$ to 0.48 in $\Theta$-solvent conditions, ${ }^{37}$ and a variation of the exponent between 0.59 to 0.50 is observed when temperature is varied from $10^{\circ} \mathrm{C}$ to $90^{\circ} \mathrm{C}, 38$ approaching the $L C S T$ of PEO (near $100^{\circ} \mathrm{C}$ ). Therefore the exponent around 0.49 observed in this work for diblock ELPs unimers at $15^{\circ} \mathrm{C}$ makes sense, as these thermosensitive chains are in a short temperature range below their $C M T$ (from $11^{\circ} \mathrm{C}$ for the less hydrated ELP200-60 to $27^{\circ} \mathrm{C}$ for the most hydrophilic ELP40-60).

\section{Studies of diblock ELPs in the nanoparticle regime}

In order to study the self-assembly of diblock ELPs at temperatures above their CMT, as well as the structural characteristics in this nanoparticle regime, solutions of diblock ELPs at a concentration of $10 \mathrm{mg} \cdot \mathrm{mL}^{-1}$ in $\mathrm{D}_{2} \mathrm{O}$ were analyzed from $15^{\circ} \mathrm{C}$ to $50^{\circ} \mathrm{C}$. Typical SANS curves are plotted in Figure 4. 


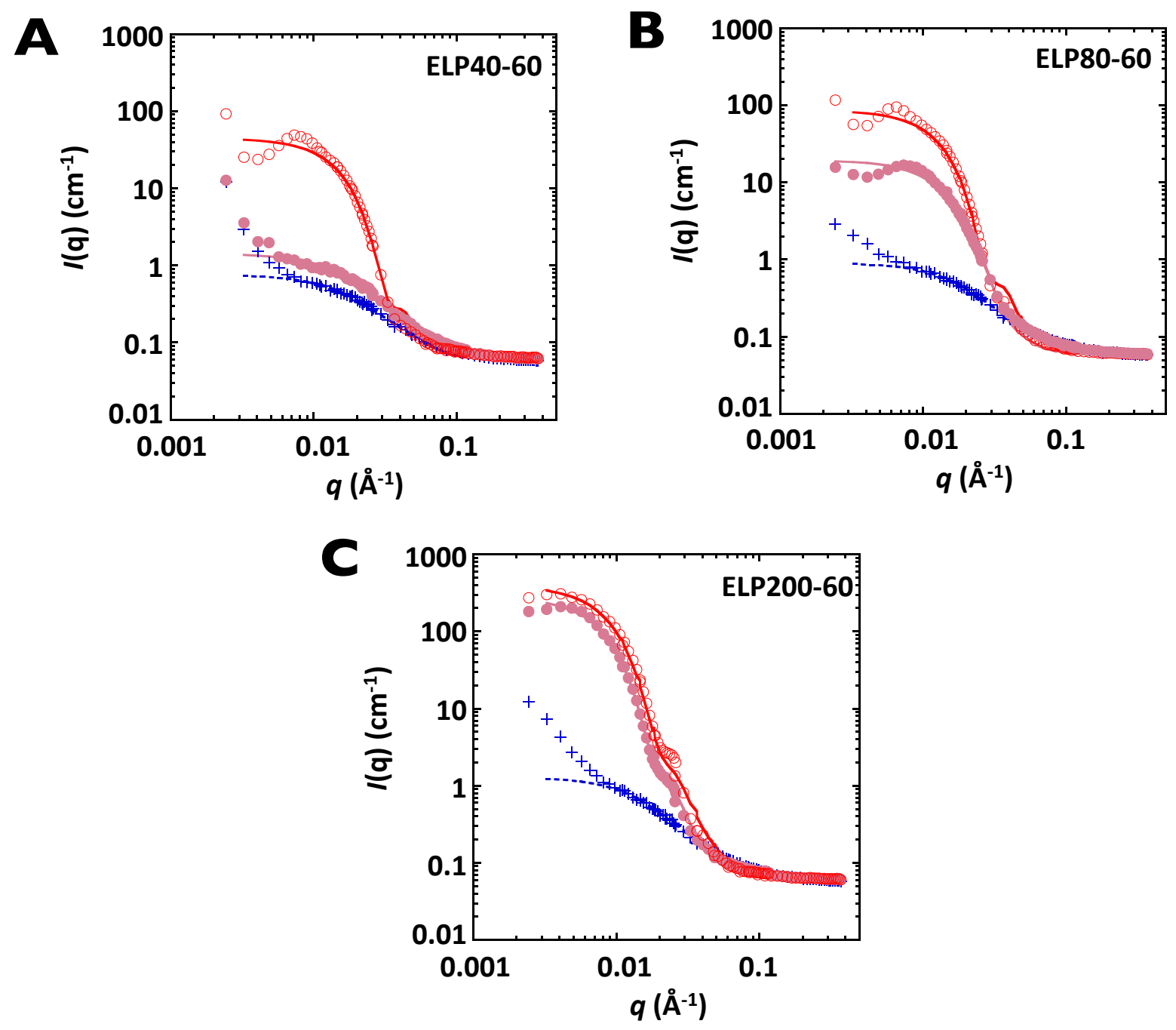

Figure 4. SANS curves of diblock ELPs 40-60 (A), 80-60 (B) and 200-60 (C) in $\mathrm{D}_{2} \mathrm{O}$ at $15^{\circ} \mathrm{C}(+), 30^{\circ} \mathrm{C}(\bullet)$ and $50^{\circ} \mathrm{C}(\mathrm{o})$. Dotted blue lines and full red lines are the best fits to the Debye function and to the hard-sphere model, respectively.

At $15^{\circ} \mathrm{C}$, as both hydrophobic and hydrophilic ELP blocks are solvated, the scattering curves were fit with the Debye function for dissolved chains similarly to the experiment previously performed under dilute conditions (vide supra). However, the fitted $R_{\mathrm{G}}$ values were found slightly smaller than previously. (Table 4) Such a decrease of the apparent $R_{\mathrm{G}}$ of ELP chains can be attributed to the attractive interactions mentioned previously, which are stronger at higher concentration. This "macromolecular crowding" phenomenon has previously been described for concentrated solutions of synthetic polymer ${ }^{39}$ and proteins. 
A significant increase of the scattering intensities by more than two orders of magnitude was observed with increasing temperature. For all diblock ELPs at $50^{\circ} \mathrm{C}$, a clear oscillation was seen in the range $0.02-0.04 \AA^{-1}$, characteristic of the form factor of monodisperse spherical objects. Except for ELP120-60 (Figure S14), the scattering curves at low $q$ did not reach a plateau as expected in the Guinier regime for nanometer sized-objects $\left(q \cdot R_{\mathrm{G}}<1\right)$. Instead, bumps near 0.007-0.009 $\AA^{-1}$ as well as a weak upturn at very low $q$ were observed. These two features indicate a combination of both repulsive and attractive interactions respectively, due to the contribution of an interparticle structure factor $S_{\text {intra }}(q) .{ }^{40}$ Scattering curves showing a weak oscillation at large $q$ were not well-fit with the simple sphere model. Fits to the hard-sphere (HS) model were of much better quality, especially in the low $q$ range. For these HS fits, aggregates were assumed to contain a certain number of entrapped $\mathrm{D}_{2} \mathrm{O}$ molecules, reducing the contrast with the solvent. The hard-sphere fraction was pinned to the experimental value $\eta_{\mathrm{HS}}=\Phi$ and the neutron SLD of aggregates $\rho_{\text {Agg }}$ was allowed to vary. This fitting method allowed us to calculate the volume fraction of $\mathrm{D}_{2} \mathrm{O}$ molecules $\left(\phi_{\mathrm{D}_{2} \mathrm{O}}\right)$ in the aggregates by:

$$
\phi_{\mathrm{D}_{2} \mathrm{O}}=\left(\rho_{\text {Agg }}-\rho_{\mathrm{ELP}_{\text {theo }}}\right) /\left(\rho_{\mathrm{D}_{2} \mathrm{O}}-\rho_{\mathrm{ELP}_{\text {theo }}}\right)
$$

Eq. 11

Aggregation numbers defined as the number of ELP chains per sphere were then estimated using the following equation:

$$
N_{\text {Agg. }}=\frac{d \cdot N_{\mathrm{A}}}{M_{\mathrm{w}}}\left(1-\phi_{\mathrm{D}_{2} \mathrm{O}}\right)\left(\frac{4 \pi R_{\mathrm{HS}}^{3}}{3}\right)
$$

Eq. 12

where $d$ and $M_{\mathrm{w}}$ are respectively the mass density and molecular weight of ELPs, $R_{\mathrm{HS}}$ the hard-sphere core size from the fit, and $N_{\mathrm{A}}$ the Avogadro number. All these parameters are listed in Table 4. In order to determine the degree of hydration of the diblock ELPs in these nanoparticles, one can also derive the average numbers of $\mathrm{D}_{2} \mathrm{O}$ molecules per VPGXG motif in micelles from these volume fractions using the number of pentapeptides in each ELP, the aggregation number and the molecular volume, $v=30 \AA^{3}$ for $\mathrm{D}_{2} \mathrm{O}$ :

$$
N_{/ \mathrm{VPGXG}}^{\mathrm{D}_{2} \mathrm{O}}=\frac{\phi_{\mathrm{D}_{2} \mathrm{O}}}{N_{\mathrm{Agg} .} \cdot N_{/ \mathrm{ELP}}^{\mathrm{VPGXG}} \cdot v_{\mathrm{D}_{2} \mathrm{O}}}\left(\frac{4 \pi R_{\mathrm{HS}}^{3}}{3}\right)
$$

Eq. 13 
Table 4. Best fit parameters using either the Debye function or the hard-sphere model from $15^{\circ} \mathrm{C}$ to $50^{\circ} \mathrm{C}$.

\begin{tabular}{|c|c|c|c|c|c|c|c|c|c|c|}
\hline \multirow{2}{*}{ ELPn-m } & \multirow{2}{*}{$\begin{array}{c}c^{a} \\
\left(\mathrm{~g} \cdot \mathrm{cm}^{-3}\right)\end{array}$} & \multirow{2}{*}{$\Phi$} & \multirow{2}{*}{$\begin{array}{c}T \\
\left({ }^{\circ} \mathrm{C}\right)\end{array}$} & Debye & \multicolumn{5}{|c|}{ Hard-sphere model ${ }^{b}$} & \multirow[b]{2}{*}{$N_{/ \mathrm{VPGXG}}^{\mathrm{D}_{2} \mathrm{O}}$} \\
\hline & & & & $\begin{array}{c}R_{\mathrm{G}} \\
(\mathrm{nm})\end{array}$ & $\begin{array}{c}R_{\mathrm{S}} \\
(\mathrm{nm})\end{array}$ & $\sigma_{\mathrm{LN}} R_{\mathrm{S}}$ & $\rho_{\mathrm{Agg}}\left(\mathrm{cm}^{-2}\right)$ & $\begin{array}{c}\phi_{\mathrm{D}_{2} \mathrm{O}} \\
(\%) \\
\end{array}$ & $N_{\text {Agg. }}$ & \\
\hline \multirow{6}{*}{$40-60$} & \multirow{6}{*}{0.00997} & \multirow{6}{*}{0.00739} & 15 & 8.7 & - & - & & - & - & - \\
\hline & & & 30 & & 2.1 & 0.495 & $5.05 \times 10^{10}$ & 70.4 & 0.2 & 38 \\
\hline & & & 35 & & 8.2 & 0.288 & $4.61 \times 10^{10}$ & 60.7 & 19 & 25 \\
\hline & & & 40 & & 11.5 & 0.175 & $4.32 \times 10^{10}$ & 54.4 & 59 & 19 \\
\hline & & & 45 & & 12.3 & 0.169 & $4.09 \times 10^{10}$ & 49.3 & 81 & 16 \\
\hline & & & 50 & & 13.1 & 0.137 & $4.06 \times 10^{10}$ & 48.7 & 99 & 15 \\
\hline \multirow{6}{*}{$60-60$} & \multirow{6}{*}{0.01018} & \multirow{6}{*}{0.00754} & 15 & 10.5 & - & - & - & - & - & - \\
\hline & & & 30 & & 3 & 0.537 & $5.10 \times 10^{10}$ & 71.6 & 0.5 & 41 \\
\hline & & & 35 & & 13.1 & 0.198 & $4.32 \times 10^{10}$ & 54.6 & 73 & 19 \\
\hline & & & 40 & & 14.8 & 0.166 & $4.12 \times 10^{10}$ & 50.2 & 115 & 16 \\
\hline & & & 45 & & 15 & 0.157 & $3.97 \times 10^{10}$ & 46.9 & 127 & 14 \\
\hline & & & 50 & & 15.2 & 0.145 & $3.86 \times 10^{10}$ & 44.5 & 138 & 13 \\
\hline \multirow{6}{*}{$80-60$} & \multirow{6}{*}{0.00985} & \multirow{6}{*}{0.00730} & 15 & 9.1 & - & - & - & - & - & - \\
\hline & & & 30 & & 11 & 0.24 & $4.80 \times 10^{10}$ & 65.2 & 28 & 30 \\
\hline & & & 35 & & 14.8 & 0.14 & $4.24 \times 10^{10}$ & 53.0 & 92 & 18 \\
\hline & & & 40 & & 15.5 & 0.144 & $3.92 \times 10^{10}$ & 46.1 & 122 & 14 \\
\hline & & & 45 & & 15.2 & 0.14 & $3.84 \times 10^{10}$ & 44.3 & 119 & 13 \\
\hline & & & 50 & & 15.2 & 0.142 & $3.76 \times 10^{10}$ & 42.6 & 122 & 12 \\
\hline \multirow{6}{*}{$120-60$} & \multirow{6}{*}{0.01015} & \multirow{6}{*}{0.00752} & 15 & 10.1 & - & - & - & - & - & - \\
\hline & & & 30 & & 16.4 & 0.18 & $4.89 \times 10^{10}$ & 67.3 & 68 & 34 \\
\hline & & & 35 & & 17.2 & 0.16 & $4.00 \times 10^{10}$ & 48.1 & 124 & 15 \\
\hline & & & 40 & & 16 & 0.157 & $3.83 \times 10^{10}$ & 44.4 & 107 & 13 \\
\hline & & & 45 & & 16 & 0.147 & $3.71 \times 10^{10}$ & 41.8 & 112 & 12 \\
\hline & & & 50 & & 15.9 & 0.147 & $3.65 \times 10^{10}$ & 40.5 & 112 & 11 \\
\hline \multirow{6}{*}{$200-60$} & \multirow{6}{*}{0.01019} & \multirow{6}{*}{0.00755} & 15 & 11.6 & - & - & - & - & - & - \\
\hline & & & 30 & & 22.8 & 0.178 & $4.13 \times 10^{10}$ & 51.1 & 188 & 17 \\
\hline & & & 35 & & 21.3 & 0.179 & $3.74 \times 10^{10}$ & 42.7 & 179 & 12 \\
\hline & & & 40 & & 21.7 & 0.174 & $3.47 \times 10^{10}$ & 36.9 & 209 & 10 \\
\hline & & & 45 & & 21.2 & 0.178 & $3.45 \times 10^{10}$ & 36.4 & 196 & 9 \\
\hline & & & 50 & & 20.8 & 0.187 & $3.38 \times 10^{10}$ & 34.9 & 189 & 9 \\
\hline
\end{tabular}

${ }^{a}$ Concentrations of diblock ELP solutions were measured by gravimetry; ${ }^{b}$ The SLD of ELP nanoparticles was a fit value $\left(\rho_{\mathrm{Agg}}\right)$. The hard-sphere volume $\eta_{\mathrm{HS}}$ fraction was fixed to the experimental volume fraction $\Phi$ deduced from the concentrations $c$ and the mass density $d=1.35 \mathrm{~g} \mathrm{~cm}^{-3}$. 
This fitting method leads to $30-40 \mathrm{D}_{2} \mathrm{O}$ molecules per pentapeptide $\left(N_{/ \mathrm{VPGXG}}^{\mathrm{D}_{2} \mathrm{O}}\right)$ at $30^{\circ} \mathrm{C}$ (Table 4), a number that is significantly larger than the estimate of one $\mathrm{D}_{2} \mathrm{O}$ per pentapeptide at $15^{\circ} \mathrm{C}$. This finding is interpreted by the difference of the two structures: for single ELP chains, only the bound water molecules are probed by neutrons, whereas above CMT when ELPs are self-assembled into spherical micelles, all the water molecules entrapped within the micelles participate to lower the contrast. At the highest temperature studied $\left(50^{\circ} \mathrm{C}\right)$, the hydration level decreases to $N_{/ \mathrm{VPGXG}}^{\mathrm{D}_{2} \mathrm{O}} \sim 10$ water molecules inside the dense state of the micelles (slightly more for ELPs of highest CMT), corresponding to $\phi_{\mathrm{D}_{2} \mathrm{O}}=0.37$ (33 wt. \% when taking into account the mass densities). This volume fraction is two-fold lower than the value of $63 \mathrm{wt} \%$ of water $\left(\phi_{\mathrm{H}_{2} \mathrm{O}}=0.70\right)$ reported in the literature ${ }^{6,7}$ for the coacervate phase. In the present study, hydration degrees of $\phi_{\mathrm{D}_{2} \mathrm{O}} \sim 0.7$ are found only at temperatures from 5 to $10^{\circ} \mathrm{C}$ below $C M T$, as seen on Table 4 from the fitted values at $30^{\circ} \mathrm{C}$ for ELP40-60 and ELP60-60. These two highly hydrated samples also correspond to aggregation numbers below 1 and to a hard sphere radius below the $R_{\mathrm{G}}$ of the individual chains. Thus, they are more likely descriptive of ELP chains that progressively start to attract each other rather than the true nanoparticle regime. This pre-transition range will be called phase "i) self-assembly" in the following discussion of the results.

In addition, at all temperatures the values of $N_{/ V P G X G}^{\mathrm{D}_{2} \mathrm{O}}$ remain much lower than the 170 water molecules per VPGVG pentapeptide reported from calorimetric measurements, ${ }^{41}$ and which can be interpreted as an upper limit of dynamic clathrate-like structures of water molecules formed around the hydrophobic moieties of the peptide above its LCST. Such an organization of water outside the hard spheres cannot be detected by static SANS measurements, as these transiently organized water molecules have the same neutron SLD than the bulk solvent. Only dynamic measurements (e.g., ultra-fast infrared spectroscopy, ${ }^{42}$ dielectric spectroscopy, ${ }^{43}$ quasi-elastic neutron scattering, ${ }^{44,45}$ or nuclear magnetic resonance ${ }^{46}$ ) can detect the "dynamic hydration number" (of the order of 4-5 water molecules per hydrophobic carbon ${ }^{46}$ ) from their lower translational diffusion constant compared to bulk water molecules. ${ }^{47,48}$

Next, the size of the self-assembled micelles deduced from the fits was plotted as a function of temperature for the five diblock ELPs. As seen on Figure 5A, the scattering 
curves of the different ELPs are very sensitive to temperature. For the two larger molecular weights (ELP120-60 and ELP200-60), the change between $15^{\circ} \mathrm{C}$ and $30^{\circ} \mathrm{C}$ is abrupt and then the curves saturate (Figures 4C, S11D and S11E), with little variation between the curves at higher temperatures. In contrast, for the other diblocks of lower molecular weight and higher $C M T$, the curves evolve much more progressively (Figures 4A, 4B, 4C, S11A, S11B and S11C). Nevertheless, the hard-sphere model with adjustable SLD contrast enables fitting the data for all the samples over the complete range of temperatures. As seen in Figure S12, the resulting parameters listed in Table 4 can be interpreted by a two step-process, respectively below and above $C M T$. The first phase (i) self-assembly) could be studied only for the two most hydrophilic block ELP40-60 and ELP40-60, as their CMT is well above room temperature.

In the nanoparticle regime, the behavior can be interpreted for all the samples by a progressive densification of the hard spheres used for modeling. In this post-transition phase called "ii) densification", the water volume fraction $\phi_{\mathrm{D}_{2} \mathrm{O}}$ that starts decreasing with temperature from the previous $i$ ) self-assembly phase continues to decrease above $C M T$. In addition, the radius $R_{\mathrm{S}}$ reaches a maximum around $5^{\circ} \mathrm{C}$ above $C M T$, and then starts to collapse slightly. In contrast, $N_{\text {Agg. }}$ values remain approximately constant: $N_{\text {Agg. }} \sim 121 \pm 2$ for ELP80-60 at $T>40^{\circ} \mathrm{C}, N_{\text {Agg. }} \sim 114 \pm 7$ for ELP120-60 at $T>35^{\circ} \mathrm{C}$, and $N_{\text {Agg. }} \sim 192 \pm 11$ for ELP200-60 at $T>30^{\circ} \mathrm{C}$. This suggests that for temperatures ranging from $5^{\circ} \mathrm{C}$ to $10^{\circ} \mathrm{C}$ above the $C M T$ and up to the $L C S T$, no additional ELP molecules are incorporated into the nanoparticle, but that water molecules are expelled from the nanoparticles. For the lower $M_{\mathrm{w}}$ ELPs (ELP40-60, ELP60-60 and ELP80-60), the hard-sphere radii $R_{\mathrm{S}}$ and the numbers of diblock ELP chains per micelle $N_{\text {Agg. }}$ both increase monotonically with temperature until they saturate when the solution temperature reaches the CMT. In order to highlight a universal scenario representing the same features for all diblock ELPs, a master curve was drawn by normalizing the hard-sphere radius $R_{\mathrm{S}}$ of micelles at a given temperature $T$ by the radius of gyration $R_{\mathrm{G}}$ of ELP unimers at $15^{\circ} \mathrm{C}$ and subtracting $C M T$ from the solution temperature. As seen on Figure 5B, this representation clearly illustrates a two stepmechanism of the aggregation process that applies to all the diblock ELPs studied herein: $i$ ) the self-assembly of ELP chains starting a few ${ }^{\circ} \mathrm{C}$ below CMT, with progressively more and more chains participating in the build-up of nanoparticles; ii) 
once the CMT is reached, no additional ELP chains are available in solution to be incorporated into the micelles, but the micelles continue to evolve by expelling water. The densification of micelles is the cause of the increase of the scattered intensity above CMT observed both by neutrons and light scattering.
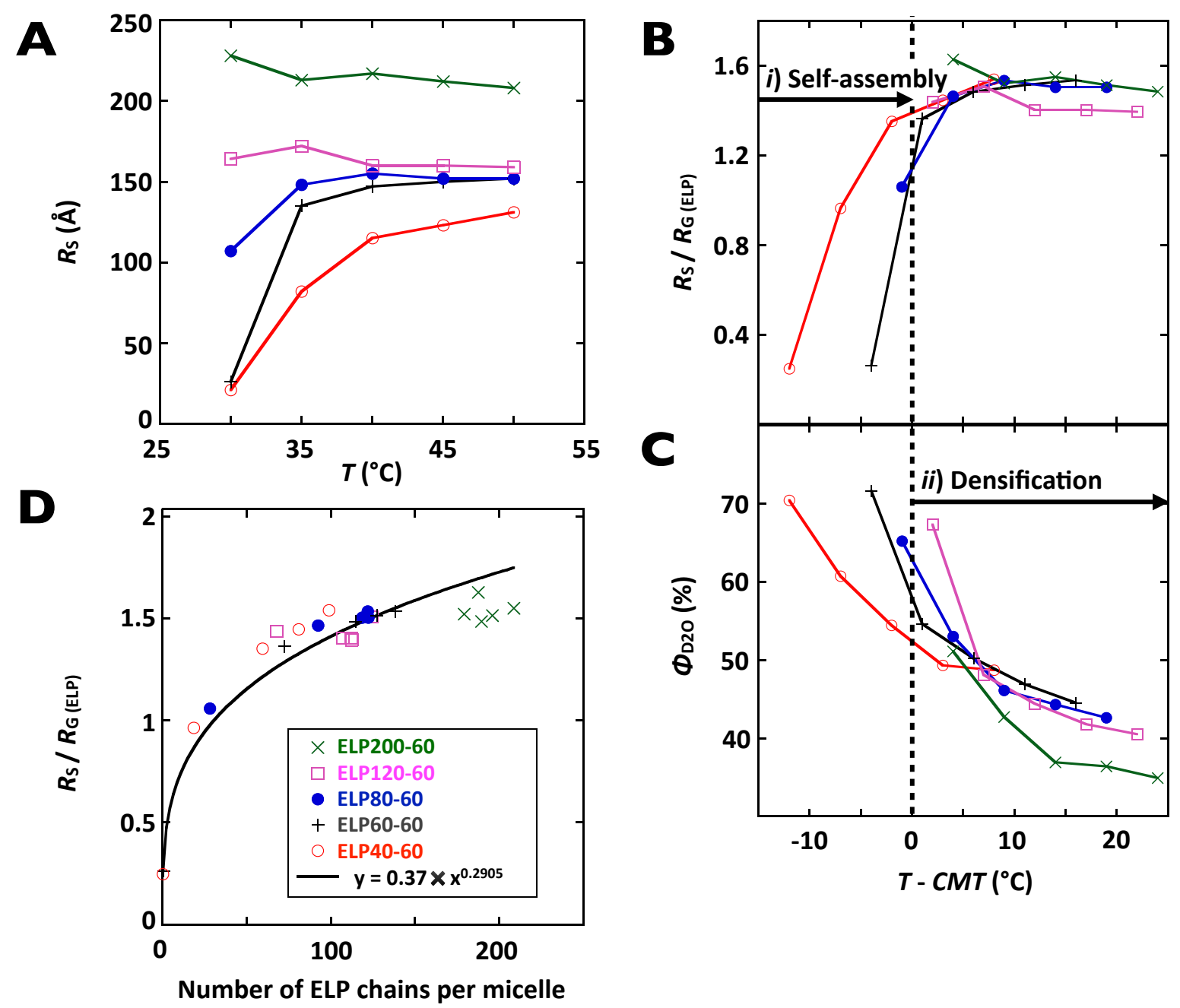

Figure 5. A) Variation of hard-sphere radii of diblock ELP nanoparticles with increasing temperature for the series of diblock ELPs 40-60 (o), 60-60 (+), 80-60 (•), 120-60(ם), and 200-60 (x). B) Master curve obtained by normalizing the hard-sphere radius by the gyration radius of the diluted ELP chains at $15^{\circ} \mathrm{C}\left(R_{\mathrm{S}} / R_{\mathrm{G}}\right)$ and by subtracting the $C M T$ measured independently by turbidimetry to temperature (T-CMT). This representation highlights the onset of the aggregation process at the CMT. C) Internal water volume fraction $\phi_{\mathrm{D}_{2} \mathrm{O}}$ in the micelles deduced from the neutron scattering contrast of the hardsphere fits plotted versus same temperature distance to CMT, showing the progressive densification of the micelles at $T>C M T$ through a continuous dehydration process 
starting below CMT and continuing above it. D) Scaling law of the micelle hard-sphere radius normalized by the gyration radius of the diluted ELP chains at $15^{\circ} \mathrm{C}\left(R_{\mathrm{S}} / R_{\mathrm{G}}\right)$ and plotted versus the number of ELP chains in the micelles $\left(N_{\mathrm{Agg}}\right)$. The solid line is a fit by a power law.

Another universal feature is the plateau value for the ratio of the hard-sphere radius of the micelles to the gyration radius of unimers that tends to a constant value: $R_{\mathrm{S}}(T>C M T) / R_{\mathrm{G}}(T \ll C M T) \approx 3 / 2$. This phenomenological ratio may appear too small at first glance, as the micelles contain up to a few hundreds ELP molecules in their cores, but one has to remember that the hydrophobic block of the chains is collapsed above CMT so that $R_{\mathrm{G}}$ scales with $M_{\mathrm{w}}$ with an exponent of $1 / 3$ instead of $1 / 2.49$. Moreover, one can compare these results with the common models of the self-assembly of amphiphilic diblock A-B copolymers, namely the "crew-cut" and the "star-like" micelle models.50 After normalizing the hard sphere radius of the micelles by the radius of gyration of the corresponding ELP chains, this ratio follows a power law of experimental slope 0.29 as a function of the aggregation number (Figure 5D). This phenomenological value is close to the theoretical exponent $1 / 4$ predicted for the radius of a star-like copolymer consisting of $p$ ideal (Gaussian) non-interacting branches linked together (an assumption that is obviously not the case with the ELP chains). ${ }^{51}$ However, in $\Theta$-solvent, such unimolecular star-like micelle follows a scaling law $R \sim N^{1 / 2} p^{1 / 4}$ that appears to be the same as the diblock ELP micelles. In this analogy, the number $N_{\mathrm{Agg}}$ of ELPs per micelle is analogous to the number of grafted arms $(p)$, and the number of pentapeptides VPGXG - is analogous to the degree of polymerization of each linear chain $(N)$. Very recently, a new regime of "weak micelles" was proposed to describe the self-assembly of similar diblock ELPn-m studied by multi-angle static and dynamic light scattering. ${ }^{52}$ Salient features of this model are the driving of unimers-to-micelle self-assembly by the surface energy of the hydrophobic blocks, and the prediction of Gaussian conformations for both the hydrophobic block ( $n$ or $N_{\mathrm{B}}$ ) in the core and the hydrophilic block ( $m$ or $N_{\mathrm{A}}$ ) in the corona, unlike standard or "strong" block copolymer micelles where the chains in the corona are stretched like in a polymer brush. These predictions are fully consistent with the results reported in this article, in particular the scaling law of micelle size $R \sim N^{1 / 2}$ where $N=n+m$ is the total number of pentapeptides (not only those forming the hydrophobic block). Aggregation numbers measured here by SANS are also close to the 
ones reported in this reference, which were measured by static light scattering. As predicted, they increase continuously with temperature above the $C M T$, and with the hydrophobic block length $n$, but decrease with the hydrophilic size $m$. We can thus conclude that the series of ELPn- $m$ studied here, with a hydrophobic length $n=40,60,80$, 120 , and 200 of VGVPG pentapeptides and a hydrophilic length kept constant $m=60$ of VPGAG alternating with VPGGG pentapeptides, globally follows the "weak micelle" model recently described, fitting with the description of ELPs as universal class of polymer-like self-assembling peptides.

\section{ConcLusion}

This paper provides a comprehensive structural analysis of a series of diblock ELPs with different sizes that shows new and unexpected insights into the temperature-triggered self-assembly process of these diblock copolymers. Below the CMT, diblock ELP chains behave like linear polymer chains, with their radius of gyration following a power law as a function of their molecular weight with an exponent value close to 0.5 that is characteristic of Gaussian coils, indicating that ELP chains are in a $\Theta$-solvent. Circular dichroism experiments performed below and above the CMT confirmed this random coil behavior. When the solution temperature approaches the $C M T$ (5 to $10^{\circ} \mathrm{C}$ below $C M T$ ), diblock ELP chains exhibit attractive intermolecular interactions and start to selfassemble into spherical objects, and the number of ELP chains in the aggregates increase until the CMT is reached. At this temperature, the diblock ELP chains form nearly monodisperse spherical nanoparticles at thermodynamic equilibrium. Increasing the temperature past the CMT, the aggregation number of ELP chains in the micelles remains constant, but micelles continuously expel water molecules from the core whose densification is evidenced by the monotonic increase in the light and neutron scattering intensities. (Figure 6) These studies shed light on the important role of water in the thermal behavior of these diblock polypeptides and suggest a new design parameter thermally-triggered desolvation and densification of the micellar core - that can be tuned at the sequence level to control the density of self-assembled polymer nanoparticles. Interestingly, all the diblock copolymers show the same behavior when represented on a master curve, and experimental scaling laws describing the evolution of micellar size $R_{\mathrm{S}}$ versus the aggregation number $N_{\text {Agg. }}$ and the molecular weight of the 
ELPs are proposed with exponents close to $1 / 4$ and $1 / 2$, respectively, similarly to synthetic amphiphilic diblock copolymers.

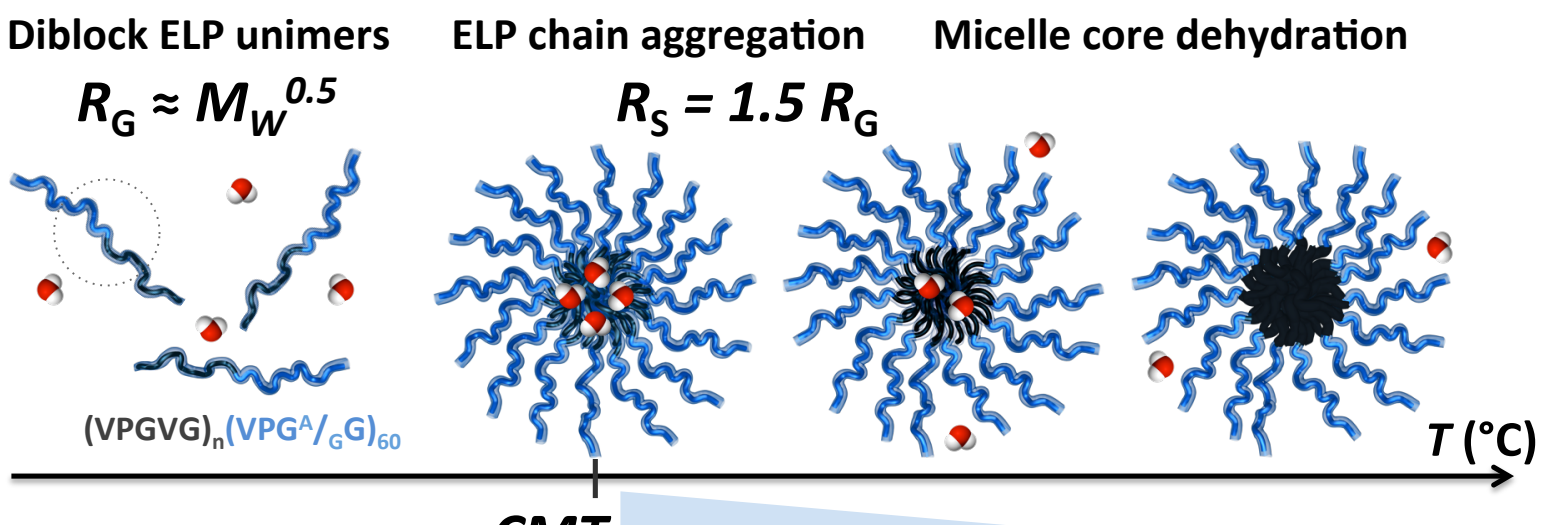

\section{CMT Water content}

Figure 6. Proposed self-assembly mechanism.

\section{AUTHOR INFORMATION}

\section{Corresponding Authors}

e.garanger@iecb.u-bordeaux.fr

lecommandoux@enscbp.fr

chilkoti@duke.edu

\section{NoTES}

The authors declare no competing financial interest.

\section{ACKNOWLEDGMENTS}

This work was partially supported by grants from the NIH (R01 EB007205 and R01 EB000188) to A.C. and by the NSF through the Research Triangle MRSEC (DMR1121107). Financial support from Centre National de la Recherche Scientifique (CNRS), Université de Bordeaux and Bordeaux-INP is gratefully acknowledged. The GIS Advanced Materials in Aquitaine and Institut Carnot MIB are greatly thanked for specific fundings to E.G. and L.B, as well as the ESF "Precision Polymer Materials" program (09RNP-124, PESC). The authors wish to thank J. R. McDaniel and K. Bathany for their help with mass spectrometry analyses. 


\section{SUPPORTING INFORMATION}

Additional details for diblock ELP constructs, chemical and physico-chemical characterizations are provided in SI, together with individual light scattering data, SANS curves and CD spectra of all diblock ELPn-60. This material is available free of charge via the Internet at http://pubs.acs.org.

\section{REFERENCES}

${ }^{1}$ Rodrígues-Cabello, J. C.; Martín, L.; Alonso, M.; Arias, F. J.; Testera, A. M. Polymer 2009, 50, 5159-5169.

${ }^{2}$ Garanger, E.; Lecommandoux, S. Angew. Chem. Int. Ed. Engl. 2012, 51(13), 3060-3062.

3 Van Hest, J. C. M.; Tirrell, D. A. Chem. Commun. 2001, 1897-1904.

${ }^{4}$ a) Simnick, A. J.; Valencia, A.; Liu, R.; Chilkoti, A. ACS Nano 2010, 4(4), 2217-2227, b) Bidwell, G. L.; Raucher, D. Advanced Drug Delivery Review 2010, 62, 1486-1496, c) Hassouneh, W.; Fisher, K.; MacEwan, S. R.; Branscheid, R.; Fu, C. L.; Liu, R.; Schmidt, M.; Chilkoti, A. Biomacromolecules 2012, 13, 1598-1605.

5 a) Urry, D. W. Journal of Protein Chemistry 1988, 7(1), 1-34, b) Urry, D. W. J. Phys. Chem. B 1997, 101, 11007-11028.

6 Urry, D. W.; Trapane, T. L.; Prasad, K. U. Biopolymers 1985, 24, 2345-2356.

7 Urry, D. W. In What sustains life? Consilient mechanisms for protein-based machines and materials; New-York, Springer-Verlag, 2005.

8 Ghoorchian, A.; Holland, N. B. Biomacromolecules 2011, 12(11), 4022-4029.

${ }^{9}$ a) Meyer, D. E.; Chilkoti, A. Biomacromolecules 2002, 3, 357-367, b) Girotti, A.; Reguera, J.; Arias, F. A.; Alonso, M.; Testera, A. M.; Rodrígues-Cabello, J. C. Macromolecules 2004, 37, 3396-3400.

${ }^{10}$ Reguera, J.; Fahmi, A.; Moriarty, P.; Girotti, A.; Rodrígues-Cabello, J. C. J. Am. Chem. Soc. 2004, 126, 13212-13213.

11 a) Lee, T. A. T.; Cooper, A.; Apkarian, R. P.; Conticello, V. Advanced Materials 2000, 12(5), 1105-1110, b) Sallach, R. E.; Wei, M.; Biswas, N.; Conticello, V.; Lecommandoux, S.; Dluhy, R.; Chaikof, E. L. J. Am. Chem. Soc. 2006, 128, 12014-12019, c) Kim, W.; Thevenot, J.; Ibarboure, E.; Lecommandoux, S.; Chaikof, E. L. Angew. Chem. Int. Ed. 2010, 49, 4257-4260.

${ }^{12}$ Dreher, M.; Simnick, A. J.; Fisher, K.; Smith, R. J.; Patel, A.; Schmidt, M.; Chilkoti, A. J. Am. Chem. Soc. 2008, 130, 687-694.

${ }^{13}$ Ribeiro, A.; Arias, F. J.; Reguera, J.; Alonso, M.; Rodrígues-Cabello, J. C. Biophysical Journal 2009, 97, 312-320.

${ }^{14}$ Aseyev, V.; Tenhu, H.; Winnik, F. M. Adv. Polym. Sci. 2011, 242, 29-89.

${ }^{15}$ Israelachvili, J. N. In Intermolecular and Surface Forces; Elsevier: London, 2011; Vol. 3. 
16 Castiglione-Morelli, A.; Scopa, A.; Tamburro, A. M.; Guantieri, V. Int. J. Biol. Macromol. 1990, 12, 363-368.

17 Urry, D. W. J. Protein Chem. 1988, 7(1), 1-34.

18 Kurzbach, D.; Hassouneh, W.; McDaniel, J.R.; Jaumann, E.A.; Chilkoti, A.; Hinderberger D. J. Am. Chem. Soc. 2013, 135(30), 11299-11308.

${ }^{19}$ McDaniel, J. R.; MacKay, J. A.; García Quiroz, F.; Chilkoti, A. Biomacromolecules 2010, 11, 944-952.

${ }^{20}$ Meyer, D. E.; Chilkoti, A. Nat. Biotechnol. 1999, 17, 1112-1115.

${ }^{21}$ McPherson, D. T.; Xu, J.; Urry, D. W. Protein Expr Purif 1996, 7, 51-57.

22 Kaye, W.; McDaniel, J. B. Appl. Optics 1974, 13, 1934-1937.

23 Pedersen, J. S. Adv. Colloid Interface Sci. 1997, 70, 171-210.

${ }^{24}$ Rayleigh, Lord Proc. Roy. Soc. London, Ser. A 1911, 84, 25-46.

25 Debye, P. J. Phys. Colloid Chem. 1947, 51, 18-32.

${ }^{26}$ Percus, J. K., Yevick, J. Phys. Rev. 1958, 110, 1-13.

27 Kinning, D.J.; Thomas, E.L. Macromolecules 1984, 17, 1712-1718.

${ }^{28}$ Baxter, R. J. J. Chem. Phys. 1968, 49, 2770-2774.

${ }^{29}$ Menon, S. V. G., Manohar, C. Srinivasa Rao, K. J. Chem. Phys. 1991, 95, 9186-9190.

30 Drappier, C.; Oliveira, H.; Sandre, O.; Ibarboure, E.; Combet; S.; Garanger, E.; Lecommandoux, S. Faraday Discussions 2013, 166, 83-100.

31 We attempted to reach lower hydrophilic weight fractions, down to $12 \%$ by using the largest hydrophobic block (VPGVG) 200 together with a twice smaller hydrophilic block with 30 repeats instead of 60, but the resulting diblock ELP200-30 did not exhibit temperature triggered self-assembly into micelles and behaved as a slubby unimer with a unique $L C S T$ around $24^{\circ} \mathrm{C}$.

32 Joanny, J.-F.; Benoît, H. Macromolecules 1997, 30, 3704-3707.

33 Wang, J. Macromolecules 2015, 48, 1614-1620.

34 Andersson, K.M.; Hovmöller, S. Z. Kristallogr. 1998, 213, 369-373.

35 Ghoorchian, A.; Simon, J. R.; Bharti, B.; Han, W.; Zhao, X.; Chilkoti , A.; López G. P. Adv. Funct. Mater. 2015, 25, 3122-3130.

36 Devenand, K.; Selser, J. C. Macromolecules 1991, 24, 5943-5947.

37 Le Coeur, C.; Teixeira, J.; Busch, P.; Longeville, S. Phys. Rev. E 2010, 81, 8061914.

38 Hammouda, B.; Ho, D. L.; Kline S. Macromolecules 2004, 37, 6932-6937.

${ }^{39}$ Le Coeur, C.; Demé, B.; Longeville, S. Phys. Rev. E 2009, 79, 031910.

40 Fresnais, J.; Berret, J-F.; Qi, L.; Chapel, J.-P.; Castaing, J.-C.; Sandre, O.; Frka-Petesic, B.; Perzynski, R.; Oberdisse, J.; Cousin, F. Phys. Rev. E 2008, 78, 040401.

${ }^{41}$ Rodríguez-Cabello, J. C.; Alonso, M.; Pérez, T.; Mar Herguedas, M. Biopolymers 2000, $54,282-288$.

42 Bakker, H. J.; Skinner, J. L. Chem. Rev. 2010, 110, 1498-1517.

43 Urry, D. W.; Peng, S. Q.; Xu, J.; McPherson, D. T. J. Am. Chem. Soc. 1997, 119, 11611162. 
44 Teixeira, J.; Bellissent-Funel, M.-C.; Chen, S. H.; Dianoux, A. J. Phys. Rev. A 1985, 31, 1913-1917.

45 Russo, D.; Murarka, R. K.; Copley, J. R. D.; Head-Gordon, T. J. Phys. Chem. B 2005, 109, 12966-12975.

46 Qvist, J. ; Halle, B. J. Am. Chem. Soc. 2008, 130, 10345-10353.

47 Laage, D.; Stirnemann, G.; Hynes, J. T. J. Phys. Chem. B 2009, 113, 2428-2435.

${ }^{48}$ Laage, D.; Stirnemann, G. Sterpone, F.; Rey, R.; Hynes, J. T. Annu. Rev. Phys. Chem. 2011, 62, 395-416.

49 Williams, C.; Brochard F.; Frisch, H-L. Ann. Rev. Phys. Chem. 1981, 32, 433-451.

50 Zhulina, E. B.; Borisov, O. V. Macromolecules 2002, 35, 9191-9203.

${ }^{51}$ Borisov, O. V.; Zhulina, E. B.; Leermakers, F. A. M.; Ballauff, M.; Müller, A. H.E Adv. Polym. Sci. 2011, 241, 1-55.

${ }^{52}$ Hassouneh, W.; Zhulina, E. B.; Chilkoti, A.; Rubinstein, M. Macromolecules 2015, 48, 4183-4195. 
For TABle OF CONTENTS USE ONLY

Structural Evolution of a Stimulus-responsive Diblock Polypeptide Micelle by Temperature Tunable Compaction of its Core

Elisabeth Garanger,* Sarah R. MacEwan, Olivier Sandre, Annie Brûlet, Laure Bataille, Ashutosh Chilkoti,* Sébastien Lecommandoux*

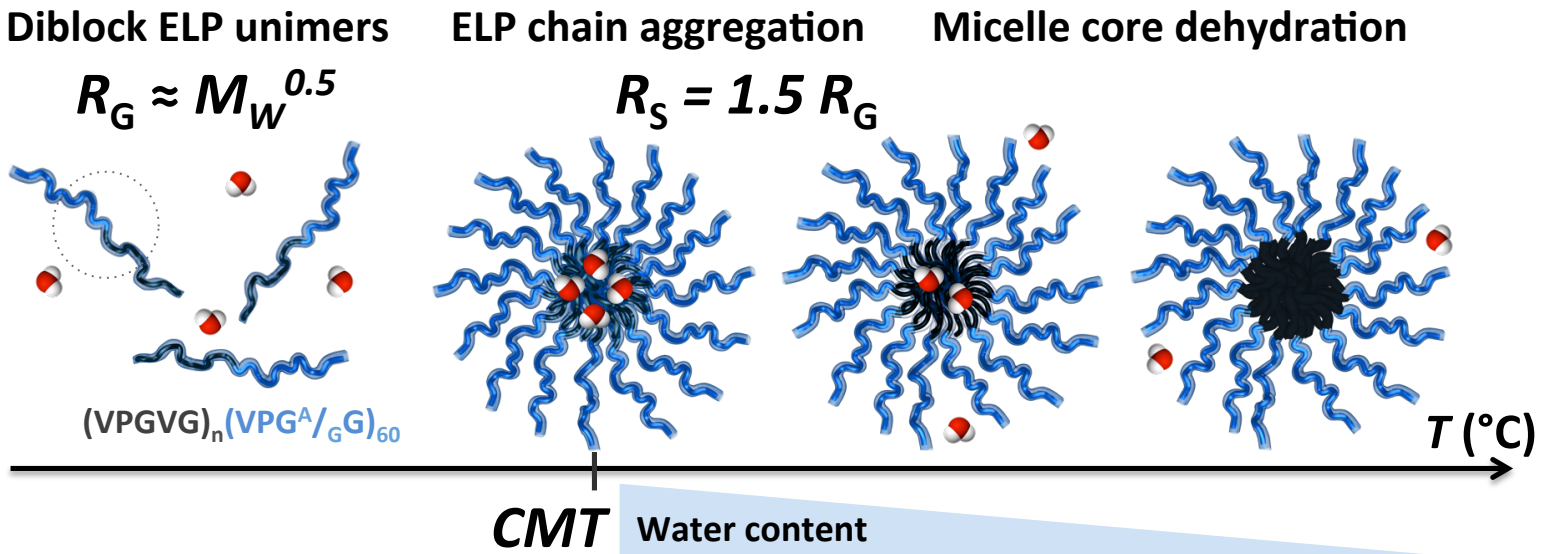

